Background Hypertrophic cardiomyopathy (HCM) is a common inherited cardiac condition. Multiple factors have been identified towards high risk of sudden cardiac death (SCD) as outlined in the ESC guidelines. $\mathrm{LV}$ wall thickness $\left(\mathrm{LV}_{\mathrm{wt}}\right)$ is an identified risk. Left ventricular mass indexed to body surface area $\left(\mathrm{LV}_{\mathrm{IBSA}}\right)$ increases with wall thickness and may predict risk not currently utilised in the risk score.

Objectives 1. Compare differences in $\mathrm{LV}_{\mathrm{wt}}$ using transthoracic echocardiogram(TTE) and MRI and the effect on ESC risk score.

2. Observe for association of $\operatorname{LV}_{\text {IBSA }}$ and incidence of nonsustained ventricular tachycardia(NSVT) either on 24 hour holter monitor or following ICD insertion.

Methods We retrospectively reviewed patients between January 2010 to July 2015 who were confirmed to have HCM on MRI. ESC Risk was calculated using LV TTE and MRI LV ${ }_{w t}$. $L_{\text {IBSA }}$ was calculated from MRI images and compared with incidence of VA on holter monitors. Patients who received ICD had follow up and VA incidence recorded.

Results 103 patients with confirmed HCM were identified with median age of 60 (range 15-87). Non sustained VT (NSVT) was recorded in $20(19.4 \%)$ patients while 16 patients had missing or no record of holter. Primary prevention ICDs (ICD ${ }_{1 *}$ ) were inserted in 20 (19.4\%) patients. MRI identified a higher absolute $\mathrm{LV}_{\mathrm{wt}}$ compared to TTE in $68.9 \%$ of patients. This lead to an increase in ESC risk score from low risk to high risk in $5 \%$ of the patients (ESC score $>4)$. LV IBSA $_{\text {Ias }}$ higher in the patients with holter positive for VA (mean $109.7 \mathrm{~g} / \mathrm{m}^{2}, 95 \%$ CI [92.8, 126.6] vs $89.8 \mathrm{~g}$ / $\mathrm{m}^{2}$, 95\% CI [83.2, 96.4], $\left.\mathrm{p}=<0.05\right)$. Patients with ICD $(n=20)$ were followed up for 52.6 months \pm 5.8 months. One patient with ICD $1^{*}$ had VA detected after 7 months of ICD insertion and treated successfully with 1 anti-tachycardia pacing algorithm.

Conclusion The detection of HCM through use of MRI allows for earlier diagnosis of patients and provides for accurate and reproducible measurements of $L_{\mathrm{wt}}$ and $\operatorname{LV}_{\mathrm{IBSA}} \mathrm{x}$ as opposed to TTE. In our retrospective study it is suggested that higher $L_{\text {IBSA }}$ is related to increase incidence of VA. If $\mathrm{LV}_{\text {IBSA }}$ derived from MRI were applied to ESC guidelines, this could result in more patients ICD ${ }_{1 *}$. We feel this should be explored with larger studies to see if $\mathrm{LV}_{\mathrm{IBSA}}$ is an independent risk factor for SCD.

\section{THE IMPORTANCE OF CONTRACTILE RESERVE WHEN ASSESSING ASYMPTOMATIC PATIENTS WITH AORTIC STENOSIS}

${ }^{1}$ Jet van Zalen*, ${ }^{2}$ Sveeta Badiani, ${ }^{1}$ Lesley Hart, ${ }^{3}$ Gary Brickley, ${ }^{3}$ Louisa Beale, ${ }^{1}$ Andrew Marshall, ${ }^{1}$ Nikhil Patel, ${ }^{2}$ Guy Lloyd. ${ }^{1}$ East Sussex Healthcare NHS Trust; ${ }^{2}$ Barts Heart centre; ${ }^{3}$ University of Brighton

\subsection{6/heartjnl-2017-311726.131}

Asymptomatic patients may exhibit symptoms during objective exercise testing, but whether symptoms are due to the obstructivity of the valve (typified by the mean gradient) or underlying ventricular function remains unknown. While the mean gradient is an easy parameter to measure no consensus about the measurement of contractile reserve exists. Longitudinal abnormalities may occur in the presence of a normal ejection fraction and the augmentation of these parameters is poorly described. The aim of this study was to examine the echocardiographic predictors of exercise ability during cardiopulmonary exercise testing combined with stress echocardiography.

24 asymptomatic patients with moderate to severe or severe aortic stenosis and preserved ejection fraction underwent stress echocardiography with simultaneous cardiopulmonary exercise testing. The primary assessment of exercise ability was $\mathrm{VO}_{2}$ peak. Echocardiography was measured at rest and during maximal exercise (defined as RER $>1$ )

$\mathrm{VO}_{2}$ peak showed a poor relationship with conventional resting parameters of severity including peak velocity $(\mathrm{rho}=0.07 ; \mathrm{p}=\mathrm{ns})$, mean pressure gradient $(\mathrm{rho}=0.3 ; \mathrm{p}=\mathrm{ns})$, AVA $(\mathrm{rho}=0.4 ; \mathrm{p}=\mathrm{ns})$, dimensionless index $(\mathrm{rho}=0.05 ; \mathrm{p}=\mathrm{ns})$, resting systolic function (by $\mathrm{EF}(\mathrm{rho}=-0.18 ; \mathrm{p}=\mathrm{ns})$ and TDI $(\mathrm{rho}=0.39 ; \mathrm{p}=\mathrm{ns})$. During exercise systolic augmentation had a good relationship with exercise ability (rho $=0.77$; $\mathrm{p}<0.0001)$ but the relationship with exercise mean gradient was weaker $(r h o=0.57 ; p=0.005)$ and there was no relationship with exercise LVEF ( $r h o=0.18 ; \mathrm{p}=\mathrm{ns}$ ).

Longitudinal systolic function during peak exercise is the strongest predictor of exercise ability when compared to conventional measures of severity of aortic stenosis.

\section{CARDIOPULMONARY EXERCISE TESTING: DOES ETHNICITY MATTER?}

Keerthi Prakash*, Stathis Papatheodorou, Bode Ensam, Chris Miles, Saeed Azizi, Paulo Bulleros, Zephryn Fanton, Aneil Malhotra, Andrew D'Silva, Gherardo Finocchiaro, Traceey Keteepe-Arachi, Maite Tome, Nicholas Bunce, Juan-Carlos Kaski, Michael Papadakis, Sanjay Sharma. St George's, University of London

\subsection{6/heartjnl-2017-311726.132}

Introduction Hypertrophic cardiomyopathy (HCM) is the largest cause of sudden cardiac death in athletes. Differentiation between HCM and athletic cardiac adaptation is not always straightforward. Cardiopulmonary exercise testing (CPET) is useful in this context, with a peak $\mathrm{VO}_{2}>120 \%$ predicted commonly used to differentiate athletes with HCM from those with physiological left ventricular hypertrophy. This value however is derived from a predominantly white population. Differences with ethnicity have been well documented on the ECG and echocardiogram of both athletes and individuals with HCM, however, ethnic differences in their physiology have not been well investigated to date.

Purpose To assess if there is a significant difference on CPET in HCM patients of black and white ethnicity.

Methods Cardiopulmonary exercise testing data was prospectively and retrospectively analysed from a cohort of 49 sedentary HCM patients assessed in a quaternary referral centre (36 white, 38 male; aged 15-65 years). Inclusion criteria: HCM patients of black or white ethnicity, NYHA 1, resting LVOT gradient $<40 \mathrm{mmHg}$, no ICD in-situ and having completed a maximal CPET (defined as: R 1.1 and test terminated due to breathlessness/muscular fatigue). 
Results Statistical significance was assessed using the MannWhitney U test (for non-parametric data) and the Student's Ttest (for parametric data). Statistically significant differences between black and white HCM patients were noted in the $\%$ predicted peak power and $\%$ predicted peak $\mathrm{VO}_{2} / \mathrm{kg}$. (Table 1)

\begin{tabular}{|c|c|c|c|}
\hline & \multicolumn{2}{|c|}{ Mean values } & \multirow{3}{*}{$-{ }^{\mathrm{p} \text {-value }}$} \\
\hline & Black & White & \\
\hline & HCM & HCM & \\
\hline Age (years) & 45.08 & 45.47 & $0.9214^{*}$ \\
\hline BMI $\left(\mathrm{kg} / \mathrm{cm}^{2}\right)$ & 26.86 & 27.96 & $0.416^{*}$ \\
\hline Peak $R$ achieved & 1.19 & 1.20 & $0.3576^{* *}$ \\
\hline$\%$ predicted $\mathrm{VO}_{2} / \mathrm{HR}$ & 95.77 & 102.42 & $0.177^{* *}$ \\
\hline $\mathrm{VE} / \mathrm{VCO}_{2}$ slope & 31.72 & 29.43 & $0.3524^{* *}$ \\
\hline \% predicted peak power (Watts) & 80.92 & 104.89 & $0.0016^{* *}$ \\
\hline Peak V0 $2 / k g(m / s / m i n / k g)$ & 25.93 & 27.53 & $0.7114^{* *}$ \\
\hline$\%$ predicted peak $\mathrm{VO}_{2} / \mathrm{kg}(\mathrm{mls} / \mathrm{min} / \mathrm{kg})$ & 77.85 & 88.03 & $0.0434^{* *}$ \\
\hline $\begin{array}{l}\text { V02 at lactate threshold (\% of peak } \mathrm{V}_{2} / \\
\mathrm{kg} \text { ) }\end{array}$ & 58.88 & 55.66 & $0.3472^{* *}$ \\
\hline
\end{tabular}

Conclusion Black HCM patients achieve a significantly lower\% predicted peak power on CPET (24\% lower) compared with white HCM patients. Black HCM patients also demonstrate a significantly lower $\%$ predicted peak $\mathrm{VO}_{2} / \mathrm{kg}$ (10\% lower) compared to white HCM patients. Larger studies are required to corroborate these ethnic differences, however, this study suggests that the current standard cut-off of a peak $\mathrm{VO}_{2}>120 \%$ predicted may be too high for a black athlete resulting in a false positive diagnosis of HCM.

\section{INCIDENCE OF INFECTIVE ENDOCARDITIS IN UK: A MULTICENTRE RETROSPECTIVE ANALYSIS}

Joseph Stansfield*. Stepping Hill

\subsection{6/heartjnl-2017-311726.133}

Background Infective Endocarditis (IE) occurs in groups of people who are deemed high risk. Previously high-risk individuals undergoing invasive procedures were recommended prophylactic antibiotics. In 2008 NICE issued new guidance to avoid prophylactic antibiotics use.
Aim We aimed to evaluate the incidence of confirmed Infective Endocarditis in two sites across the North West region prior to and after the implementation of the 2008 guidance, including causative infective organisms, Echo findings, subsequent morbidity and mortality.

Methods OPCS-4 (Office of Population, Censuses and Surveys classification-4th Edition) system and the standard code allocated were retrospectively analysed for all admissions related to Infective Endocarditis for the years 2007-2008 and then again for the years 2012-2015.

Results The incidence of IE dramatically increased for the years after the guidance came in to effect compared to prior to the guidance. Initial data from one sites showed an increase in total numbers of cases year on year. All except 3 patients had positive blood cultures. Our data also shows that mortality remains high due to IE $(2012 \%-18 \%$ of patients died as a direct result of IE, 2013\%-38\%, 2014\%-11\%, 2015\%-21\%). Conclusions Incidence of IE has increased subsequently to the universal change in NICE guidance. Mortality associated with IE being still high. Although this is a small initial study from one centre, data from our second site is being collated to add support to our findings.

\section{SOURCES OF STREPTOCOCCAL BACTERAEMIA AND THEIR IMPLICATIONS FOR THE DIAGNOSIS OF INFECTIVE ENDOCARDITIS}

Louis Baig*, Mirza Wazir Baig, Jonathan Sandoe. Leeds Teaching Hospital Trusts

\subsection{6/heartinl-2017-311726.134}

Introduction The diagnosis of infective endocarditis (IE) is dependent on the detection of a sustained bacteraemia with multiple positive blood cultures being major criteria in the Duke nosology for IE. The interpretation of a single positive blood culture growing pathogens that could cause IE, but that do not fulfil major Duke criteria, is a common diagnostic difficulty in patients with a febrile illness. This study was designed to examine the clinical outcomes in patients with streptococcal bacteraemias and to determine the proportion of these with a final diagnosis of IE.

Methods This was a retrospective descriptive analysis of patients with streptococcal bacteraemias between SeptemberDecember 2012. IE was confirmed by a Consultant Microbiologist (JS) using the modified Duke criteria. The variables

Abstract 135 Table 1 Distribution of streptococcal species isolated in blood cultures and their aetiological role in cases of infective endocarditis according to age less than or greater than 18 years.

\begin{tabular}{|l|c|c|c|c|c|}
\hline & \multicolumn{2}{|c|}{ Age <18 } & \multicolumn{2}{c|}{ Age $\geq 18$} & Total \\
\hline & IE (\%) & Not IE (\%) & IE (\%) & Not IE (\%) & \\
\hline Streptococcus pneumoniae & $0(0)$ & $4(100)$ & $0(0)$ & $27(100)$ & 31 \\
\hline Oral streptococci & $0(0)$ & $16(100)$ & $5(12.5)$ & $35(87.5)$ & 56 \\
\hline Strep. bovis group (gallolyticus) & $0(0)$ & $0(0)$ & $0(0)$ & $1(100)$ & 1 \\
\hline Beta-haemolytic streptococci & $0(0)$ & $5(100 \%)$ & $2(12.5)$ & $14(87.5)$ & 21 \\
\hline $\begin{array}{l}\text { Strep. anginosus group } \\
\text { (anginosus/intermedius/constelatus) }\end{array}$ & $0(0)$ & $1(100 \%)$ & $0(0)$ & $2(100)$ & 3 \\
\hline
\end{tabular}

